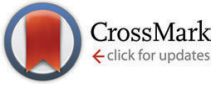

Cite this: Chem. Commun., 2016, 52,10187

Received 14th June 2016, Accepted 15th July 2016

DOI: $10.1039 / \mathrm{c} 6 \mathrm{cc} 04954 \mathrm{k}$

www.rsc.org/chemcomm

\section{A traceless photocleavable linker for the automated glycan assembly of carbohydrates with free reducing ends $\dagger$}

\author{
M. Wilsdorf, ${ }^{a}$ D. Schmidt, ${ }^{a b}$ M. P. Bartetzko, ${ }^{a b}$ P. Dallabernardina, ${ }^{a b}$ \\ F. Schuhmacher, ${ }^{a b}$ P. H. Seeberger ${ }^{a b}$ and F. Pfrengle*ab
}

\begin{abstract}
We report a traceless photocleavable linker for the automated glycan assembly of carbohydrates with free reducing ends. The reductive-labile functionality in the linker tolerates all commonly used reagents and protocols for automated glycan assembly, as demonstrated with the successful preparation of nine plant cell wall-related oligosaccharides, and is cleaved by hydrogenolysis.
\end{abstract}

Carbohydrates play a central role in materials science, biology and medicine. ${ }^{1}$ In order to study the structure-activity relationship of glycans, homogeneous and pure samples which are obtained through chemical synthesis are indispensable. Automated glycan assembly has evolved as a platform to rapidly access these complex glycans. ${ }^{2}$ The automated process assembles a diverse set of monosaccharide building blocks into oligosaccharide products without the need for manual intervention. Starting from a resin-bound linker, iterative glycosylation and deprotection steps systematically elongate the glycan chain. Photolabile linker 1 ( $c f$. Fig. 1) is often employed to link the growing glycan to the solid support. ${ }^{3}$ This linker is cleaved in a continuous flow photoreactor followed by global deprotection to release glycans containing an aminoalkyllinker at the reducing end. The amino group of the alkyl linker is utilized to attach glycans to microarray surfaces ${ }^{4}$ and carrier proteins required in the study and development of conjugate vaccine candidates. ${ }^{5}$

Naturally occurring free reducing glycans are also highly desirable, since synthetic samples are frequently needed as standards for LC/MS measurements ${ }^{6}$ or biological assays that do not tolerate the presence of unnatural modifications. To access glycans with free reducing ends by solid-phase chemistry, base-labile linker systems have been used that incorporate a

\footnotetext{
${ }^{a}$ Department of Biomolecular Systems, Max-Planck-Institute of Colloids and Interfaces, Am Mühlenberg 1, 14476 Potsdam, Germany.

E-mail: Fabian.Pfrengle@mpikg.mpg.de

${ }^{b}$ Freie Universität Berlin, Institute of Chemistry and Biochemistry, Arnimallee 22, 14195 Berlin, Germany

$\dagger$ Electronic supplementary information (ESI) available: Experimental procedures for solution-phase reactions and automated glycan assembly and characterization data for all compounds. See DOI: 10.1039/c6cc04954k
}

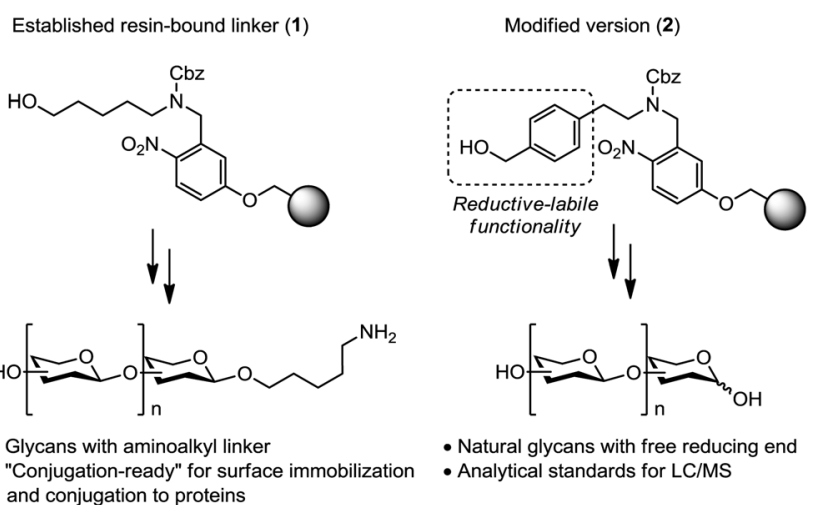

Fig. 1 Photocleavable linker 1 and traceless linker 2 for the automated assembly of glycans with an aminoalkyl linker and a free reducing end, respectively.

benzylic group to release the anomeric hydroxyl after a final hydrogenation step. ${ }^{7}$ Based on this precedence, we envisioned that the introduction of a benzyl moiety into photocleavable linker 1 would allow for rapid access to free reducing glycans after photo-induced cleavage of the glycans from the solid support followed by hydrogenolysis. Here, we report the synthesis and use of traceless linker 2, whose applicability was demonstrated in the automated assembly of various complex glycans with free reducing ends (Fig. 1).

Following the established route towards photo-cleavable linker $\mathbf{1}$, the preparation of 2 required only the substitution of the 5-aminopentanol portion for benzylic aminoalcohol 5, which is commercially available but very expensive. We designed a short and scalable route towards aminoalcohol 5 that provides an attractive alternative to the available literature procedures. ${ }^{8}$ Readily available terephthaldehyde (3) was selectively reduced once with $\mathrm{NaBH}_{4}$ and condensed with nitromethane in an Henry reaction to form nitroalkene $4^{9,10}$ Subsequent conjugate reduction of the nitroalkene with $\mathrm{NaBH}_{4}$ in DMSO gave the corresponding nitroalkane (not shown) which was further reduced to the primary amine by hydrogenation and then isolated as its 

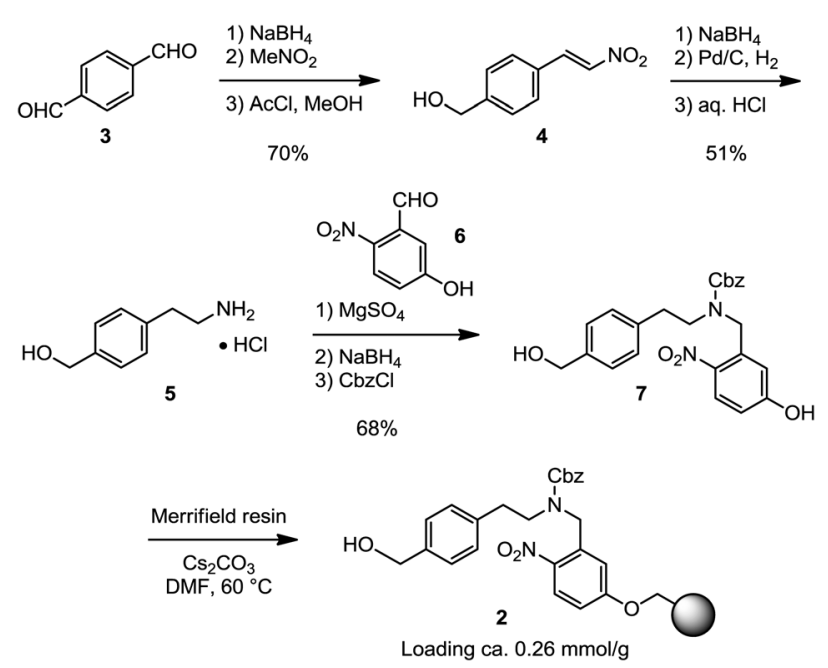

Scheme 1 Gram-scale preparation of the resin-bound linker 2.

hydrochloride salt 5. With sufficient quantities of the aminoalcohol in hand, photocleavable linker 7 was obtained by reductive amination of $\mathbf{5}$ with aryl aldehyde $\mathbf{6}$ followed by protection of the resulting secondary amine with $\mathrm{CbzCl}^{3}$ This short route was readily scaled up for the preparation of a seven gram batch of the product. Subsequent functionalization of Merrifield resin by alkylation with four equivalents of linker 7 finally provided resin-bound linker 2 with a loading determined to be approximately $0.26 \mathrm{mmol} \mathrm{g}^{-1}$ (Scheme 1). ${ }^{3,11}$ Excess linker 7 was efficiently recovered after functionalization.

The automated assembly of fully-protected cellulose tetrasaccharide $\mathbf{9}$ was selected as a proof-of-principle study for the utility of the new linker (Scheme 2). The synthesis of 9 included four iterative cycles of glycosylation and Fmoc-deprotection steps employing monosaccharide building block $\mathbf{8}$, followed
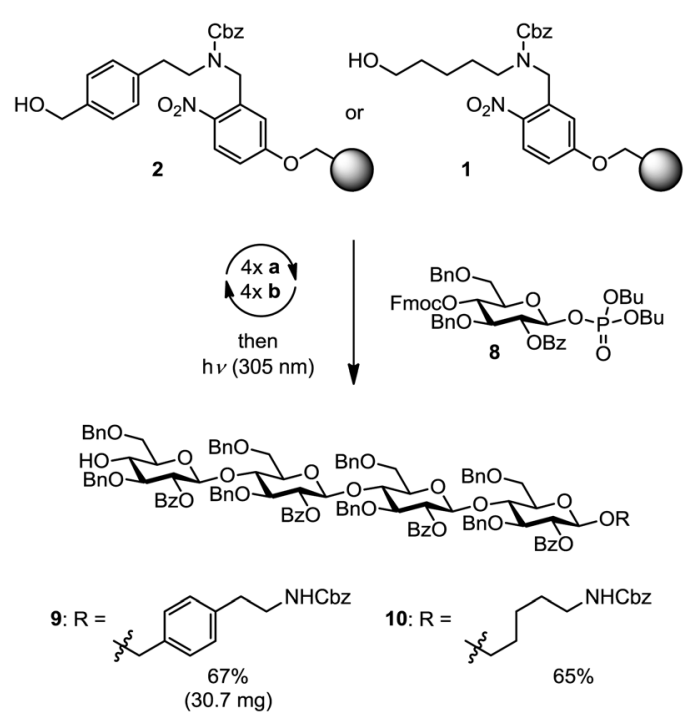

Scheme 2 Head-to-head comparison of the traceless linker 2 and the aminoalkyl linker 1. Glycosylation step (a): 3.7 equiv. of $\mathbf{8}$, TMSOTf, $\mathrm{CH}_{2} \mathrm{Cl}_{2},-35^{\circ} \mathrm{C} \rightarrow-15^{\circ} \mathrm{C}$; deprotection step (b): $20 \% \mathrm{NEt}_{3}$ in DMF, $25^{\circ} \mathrm{C}$. Yields are based on resin loading. by photolytic cleavage of the linker from the solid-support prior to final HPLC purification. Target molecule 9 was isolated in $67 \%$ yield and thus virtually with the same efficiency as aminopentylsubstituted cellulose derivative $\mathbf{1 0}$ that was obtained using linker $\mathbf{1}$. Notably, the presence of an additional benzene moiety in the linker did not affect the efficiency of the glycosylation and deprotection steps in the automated assembly as well as the subsequent photolytical cleavage. Next, cellulose tetrasaccharide 9 was sequentially deprotected by methanolysis of the benzoate esters (with NaOMe) followed by hydrogenolysis of the benzyl ethers and the linker $\left(\mathrm{H}_{2}, \mathrm{Pd} / \mathrm{C}\right)$, affording cellulose tetrasaccharide $\mathbf{1 1}$ as a mixture of $\alpha / \beta$-anomers. During concentration of the crude product, the open chain form of $\mathbf{1 1}$ reacted partially with the primary amine $\mathbf{1 2}$ to form a hemiaminal ether. ${ }^{12}$ Acidic hydrolysis converts this mixture to the final product $\mathbf{1 1}$ before it was subjected to final HPLC purification. As verified by analytical HPLC, MS and 2D NMR spectroscopy, the tetrasaccharide product was obtained in high purity, albeit in a rather low yield (20\%) over the two deprotection steps. The moderate yield may be attributed to the low solubility of the partially protected intermediate (not shown), impeding purification by reversed phase-HPLC. The deprotection sequence is currently optimized to increase the overall efficiency of the process (Scheme 3).

The utility of linker 2 was further illustrated by preparing a diverse set of plant cell wall-related oligosaccharides (Scheme 4). Seven orthogonally protected monosaccharide building blocks (BB) 8 and 13-18 were used for the automated glycan assembly of cellulose-, arabinoxylan-, and arabinogalactan-type oligosaccharides. ${ }^{13}$ Following iterative glycosylation and chemoselective deprotection steps, various linear and branched oligosaccharides up to hexasaccharides were obtained after photolytical cleavage from the solid support. These protected products were then subjected to methanolysis and hydrogenation to afford pure glycans $19-26$ in 3-18\% overall yield. For the automated assembly of the xylan oligosaccharides 20-23, the use of only 2.8 equiv. of building block per glycosylation step was sufficient, whereas for the construction of the notoriously difficult to synthesize Gal- $\beta-1,3-G a l-l i n k a g e$ (24-26) eight equiv. of building block were necessary. ${ }^{13 b}$ Linker 2 proved to be compatible with all common protocols for automated glycan assembly, including glycosylations with glycosyl phosphates and thioglycosides, the removal of protecting groups such as Fmoc,

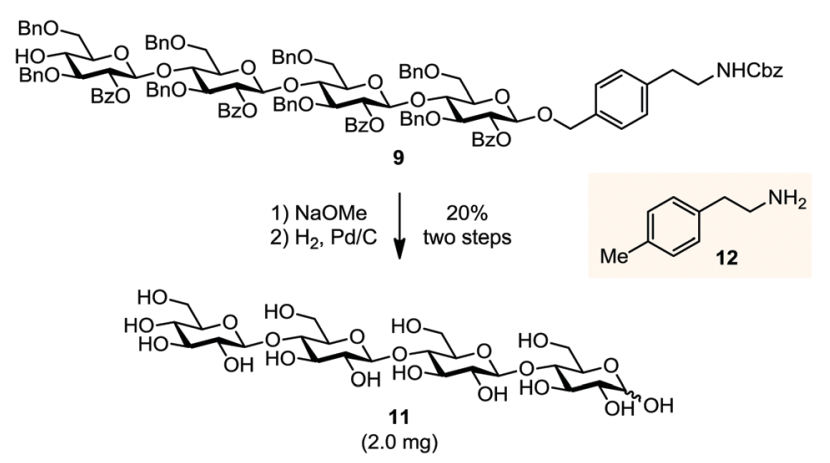

Scheme 3 Global deprotection of cellulose tetrasaccharide $\mathbf{9}$. 

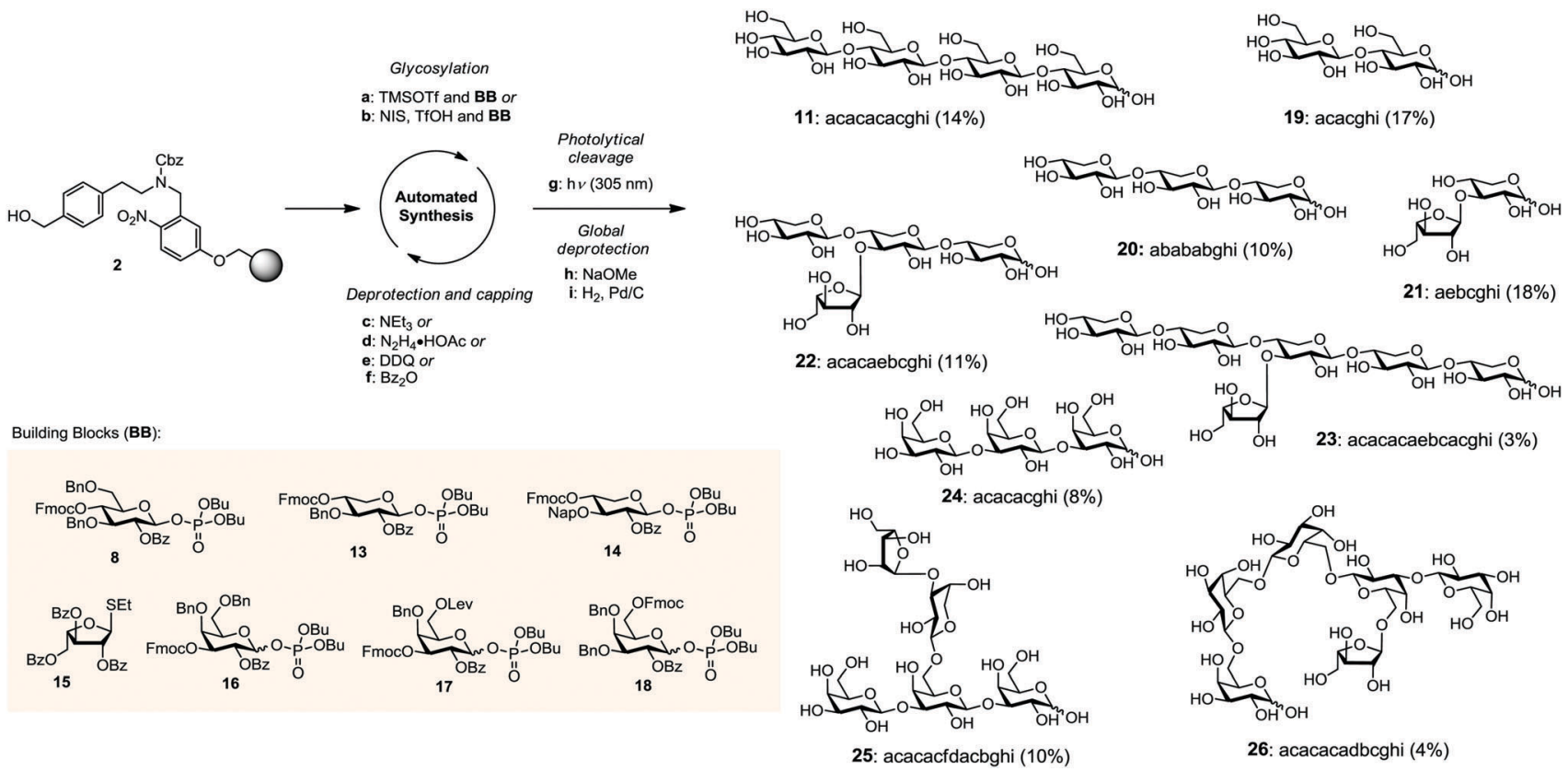

Scheme 4 Automated assembly of plant cell wall oligosaccharides 11, 19-26. Synthesizer modules (a): one or two cycles of 1.4-4.0 equiv. of BB, TMSOTf, $\mathrm{CH}_{2} \mathrm{Cl}_{2},-35{ }^{\circ} \mathrm{C}$ or $-30{ }^{\circ} \mathrm{C}$ (5 min) $\rightarrow-15{ }^{\circ} \mathrm{C}$ or $-20{ }^{\circ} \mathrm{C}(30 \mathrm{~min})$; (b): two cycles of $1.4-4.0$ equiv. of $\mathrm{BB}, \mathrm{NIS} / \mathrm{TfOH}, \mathrm{CH} \mathrm{Cl}_{2} / \mathrm{dioxane}(2: 1)$, $-40{ }^{\circ} \mathrm{C}(5 \mathrm{~min}) \rightarrow-20^{\circ} \mathrm{C}(40 \mathrm{~min}) ;(\mathrm{c}): 3$ cycles of $20 \% \mathrm{Et}_{3} \mathrm{~N}$ in DMF, $25^{\circ} \mathrm{C}(5 \mathrm{~min}) ;(\mathrm{d}): 3$ cycles of $\mathrm{N}_{2} \mathrm{H}_{4} \cdot \mathrm{HOAc}(0.15 \mathrm{M})$ in pyridine/AcOH/ $\mathrm{H}_{2} \mathrm{O}(4: 1: 0.25)$, $25^{\circ} \mathrm{C}(30 \mathrm{~min})$; (e): six cycles of DDQ $(0.10 \mathrm{M})$ in DCE/MeOH/H $\mathrm{H}_{2} \mathrm{O}(64: 16: 1), 40{ }^{\circ} \mathrm{C}(20 \mathrm{~min})$; (f): three cycles of $\mathrm{Bz} 2 \mathrm{O}(0.50 \mathrm{M}$ in DCE) and DMAP (0.25 M in DCE), pyridine, $40^{\circ} \mathrm{C}$ (30 min). Photolytical cleavage (g): $h \nu$ (305 nm), DCM, rt. Global deprotection (h): $\mathrm{NaOMe}, \mathrm{MeOH}, \mathrm{rt}(16 \mathrm{~h}) ;(\mathrm{i}): \mathrm{H} 2,10 \% \mathrm{Pd} / \mathrm{C}$, EtOAc/MeOH/H $/ \mathrm{H}_{2} \mathrm{O} / \mathrm{AcOH}$, rt $(16 \mathrm{~h})$. Yields are based on resin loading. The letter codes below the structures represent the synthesizer modules and deprotection steps applied in the respective syntheses.

Lev and Nap, as well as the capping of free hydroxyl groups as benzoyl esters.

In conclusion, we have synthesized nine plant cell wallrelated glycans using a novel traceless photocleavable linker that enables the automated glycan assembly of carbohydrates with free reducing ends. The linker complements the photocleavable linker for the synthesis of aminoalkyl-functionalized glycans and provides the oligosaccharides in comparable yields. The opportunity to produce large sets of related glycans with free reducing ends holds great potential for the preparation of standards for LC/MS- and ion mobility mass spectrometry experiments. ${ }^{6,14}$

We gratefully acknowledge financial support from the Max Planck Society, the German Research Foundation (DFG, Emmy Noether program PF850/1-1 to FP, fellowship Wi4356/2-1 to MW, and the SFB765), the Fonds der Chemischen Industrie (Liebig-fellowship to FP) and an ERC Advanced Grant (AUTOHEPARIN to PHS).

\section{Notes and references}

1 (a) N. Taniguchi, T. Endo, G. W. Hart, P. H. Seeberger and C.-H. Wong, Glycoscience: Biology and Medicine, Springer, Tokyo, 2015; (b) S. Dumitriu and V. Popa, Polymeric Biomaterials: Structure and Function, CRC Press, Boca Raton, 2013.

2 (a) O. J. Plante, E. R. Palmacci and P. H. Seeberger, Science, 2001, 291, 1523-1527; (b) L. Kröck, D. Esposito, B. Castagner, C.-C. Wang, P. Bindschädler and P. H. Seeberger, Chem. Sci., 2012, 3, 1617-1622; (c) P. H. Seeberger, Acc. Chem. Res., 2015, 48, 1450-1463.

3 (a) S. Eller, M. Collot, J. Yin, H. S. Hahm and P. H. Seeberger, Angew. Chem., Int. Ed., 2013, 52, 5858-5861; (b) O. Calin, S. Eller and
P. H. Seeberger, Angew. Chem., Int. Ed., 2013, 52, 5862-5865; (c) J. Kandasamy, F. Schuhmacher, H. S. Hahm, J. C. Klein and P. H. Seeberger, Chem. Commun., 2014, 50, 1875-1877; (d) R. J. Fair, H. S. Hahm and P. H. Seeberger, Chem. Commun., 2015, 51, 6183-6185.

4 C. R. Rillahan and J. C. Paulson, Annu. Rev. Biochem., 2011, 80, 797-823.

5 (a) Z. Guo and G.-J. Boons, Carbohydrate-Based Vaccines and Immunotherapies, John Wiley \& Sons, New Jersey, 2009; (b) F. Broecker, C. Anish and P. H. Seeberger, Methods Mol. Biol., 2015, 1331, 57-80.

6 M. P. Campbell, T. Nguyen-Khuong, C. A. Hayes, S. A. Flowers, K. Alagesan, D. Kolarich, N. H. Packer and N. G. Karlsson, Biochim. Biophys. Acta, 2014, 1844, 108-116.

7 (a) X. Wu, M. Grathwohl and R. R. Schmidt, Angew. Chem., Int. Ed., 2002, 41, 4489-4493; (b) K. Tanaka, Y. Fujii, H. Tokimoto, Y. Mori, S.-I. Tanaka, G.-M. Bao, E. R. O. Siwu, A. Nakayabu and K. Fukase, Chem. - Asian J., 2009, 4, 574-580; (c) T. J. Boltje, J.-H. Kim, J. Park and G.-J. Boons, Nat. Chem., 2010, 2, 552-557.

8 (a) L. Pignataro, S. Carboni, M. Civera, R. Colombo, U. Piarulli and C. Gennari, Angew. Chem., Int. Ed., 2010, 49, 6633-6637; (b) J.-P. Behr and J.-M. Lehn, Helv. Chim. Acta, 1980, 63, 2112-2118.

9 (a) J. M. Lopchuk, R. P. Hughes and G. W. Gribble, Org. Lett., 2013, 15, 5218-5221; (b) S. Peterli, D. Hubmann, U. Sequin, H. Mett and P. Traxler, Helv. Chim. Acta, 1994, 77, 59-69.

10 The generation of partially $O$-acetylated nitroalkene required a subsequent acidic methanolysis to afford pure 4 .

11 For loading determination, see the ESI $\dagger$.

12 W. Pigman, E. A. Cleveland, D. H. Couch and J. H. Cleveland, J. Am. Chem. Soc., 1951, 73, 1976-1979.

13 (a) D. Schmidt, F. Schuhmacher, A. Geissner, P. H. Seeberger and F. Pfrengle, Chem. - Eur. J., 2015, 21, 5709-5713; (b) M. Bartetzko, F. Schuhmacher, H. S. Hahm, P. H. Seeberger and F. Pfrengle, Org. Lett., 2015, 17, 4344-4347; (c) P. Dallabernardina, F. Schuhmacher, P. H. Seeberger and F. Pfrengle, Org. Biomol. Chem., 2016, 14, 309-313.

14 J. Hofmann, H. S. Hahm, P. H. Seeberger and K. Pagel, Nature, 2015, 526, 241-244. 